\title{
ON THE GIBBS PHENOMENON IV: RECOVERING EXPONENTIAL ACCURACY IN A SUBINTERVAL FROM A GEGENBAUER PARTIAL SUM OF A PIECEWISE ANALYTIC FUNCTION
}

\author{
DAVID GOTTLIEB AND CHI-WANG SHU
}

\begin{abstract}
We continue our investigation of overcoming the Gibbs phenomenon, i.e., to obtain exponential accuracy at all points (including at the discontinuities themselves), from the knowledge of a spectral partial sum of a discontinuous but piecewise analytic function. We show that if we are given the first $N$ Gegenbauer expansion coefficients, based on the Gegenbauer polynomials $C_{k}^{\mu}(x)$ with the weight function $\left(1-x^{2}\right)^{\mu-1 / 2}$ for any constant $\mu \geq 0$, of an $L_{1}$ function $f(x)$, we can construct an exponentially convergent approximation to the point values of $f(x)$ in any subinterval in which the function is analytic. The proof covers the cases of Chebyshev or Legendre partial sums, which are most common in applications.
\end{abstract}

\section{INTRODUCTION}

In this paper we continue our investigation of overcoming the Gibbs phenomenon, i.e., recovering pointwise exponential accuracy at all points, including at the discontinuities themselves, from the knowledge of a spectral partial sum of a discontinuous but piecewise analytic function, which we started in [4, 5 and 6$]$.

Spectral approximations, such as the Fourier approximation based upon trigonometric polynomials for periodic problems, and the Chebyshev, Legendre or the general Gegenbauer approximation based upon polynomials for nonperiodic problems, are exponentially accurate for analytic functions [3, 2]. However, for discontinuous but piecewise analytic functions, the spectral partial sum approximates the function poorly throughout the domain. Away from the discontinuity only first-order accuracy is achieved. Near the discontinuity there are $O(1)$ oscillations which do not decrease with $N$, the number of terms retained in the spectral sum. This is known as the Gibbs phenomenon.

Our framework in [4, 5 and 6] to overcome the Gibbs phenomenon and obtain exponential accuracy at all points for piecewise analytic functions relies

Received by the editor March 22, 1994 and, in revised form, June 16, 1994.

1991 Mathematics Subject Classification. Primary 42A10, 41A10, 41 A25.

Key words and phrases. Gibbs phenomenon, Gegenbauer polynomials, exponential accuracy.

Research supported by AFOSR grant 93-0090, ARO grant DAAL03-91-G-0123, DARPA grant N00014-91-J-4016, NSF grant DMS-9211820, NASA grant NAG1-1145, and NASA contract NAS119480 while the authors were in residence at ICASE, NASA Langley Research Center, Hampton, VA 23665 . 
heavily on using the Gegenbauer polynomials $C_{n}^{\lambda}(x)$, which are orthogonal in $[-1,1]$ with the weight function $\left(1-x^{2}\right)^{\lambda-\frac{1}{2}}$, for large $\lambda$. We assume that the first $-N \leq k \leq N$ Fourier coefficients, or the first $0 \leq k \leq N$ Legendre coefficients, of a discontinuous but piecewise analytic function, are given. The procedure consists of two steps:

1. Using the given spectral partial sum of the first $N$ terms, to recover the first $m \sim N$ Gegenbauer expansion coefficients, based on a subinterval $[a, b] \subset[-1,1]$ in which the function is presumably analytic, with exponential accuracy. This can be achieved for any $L_{1}$ function, as long as we choose $\lambda$ in the weight function of Gegenbauer polynomials to be proportional to $N$. The error incurred at this stage is called the truncation error.

2. For an analytic function in $[a, b]$, proving the exponential convergence of its Gegenbauer expansion, when the parameter $\lambda$ in the weight function is proportional to the number of terms retained in the expansion. The error at this stage is labeled the regularization error.

In [6] we demonstrated this procedure in the case of a discontinuous but piecewise analytic function, provided its Fourier or Legendre spectral partial sum is given.

The proof of the Legendre case in [6] is based upon first expanding the Legendre polynomial $P_{k}(x)=C_{k}^{\frac{1}{2}}(x)$ into its Fourier series:

$$
P_{k}(x)=\sum_{l=-\infty}^{\infty} a_{l}^{k} e^{i l \pi x} .
$$

It was essential in this proof that the Fourier expansion for the Legendre polynomial $P_{N}(x)$, for large $N$, contains lower terms that decay exponentially with $N$ :

$$
\left|a_{k}^{N}\right| \leq A \min \left(1,\left(\frac{e \pi k}{2 N}\right)^{N}\right) .
$$

Unfortunately, it seems that this fact is true only for Legendre polynomials, probably because their weight function is special $(\equiv 1)$. It seems not true for other Gegenbauer polynomials, such as Chebyshev polynomials. In an earlier version of [6], we quoted a formula (7.354, page 836 of [7]) to this effect for Chebyshev polynomials. However, it is doubtful that formula 7.354 of [7] is correct.

In this paper, we will consider the case of general Gegenbauer spectral methods, with Chebyshev and Legendre methods as special cases. We assume that $f(x)$ is an $L_{1}$ function on $[-1,1]$ and analytic in a subinterval $[a, b] \subset$ $[-1,1]$. We also assume that the Gegenbauer partial sum of $f(x)$, based upon the Gegenbauer polynomials $C_{k}^{\mu}(x)$ with the weight function $\left(1-x^{2}\right)^{\mu-\frac{1}{2}}$ for any constant $\mu \geq 0$, over the full interval $[-1,1]$, is known. The objective is to recover exponentially accurate point values over the subinterval $[a, b]$ of analyticity.

We will follow the same path as in [6]. Basically we will show that the first $0 \leq k \leq N$ Gegenbauer expansion coefficients, based on the Gegenbauer polynomials $C_{k}^{\mu}(x)$ for any constant $\mu \geq 0$, contain enough information such that a different, rapidly converging Gegenbauer expansion in the subinterval $[a, b]$, 
with the parameter $\lambda$ in the weight function $\left(1-\xi^{2}\right)^{\lambda-\frac{1}{2}}$ being proportional to $N$, can be constructed. As before, we will separate the analysis of the error into two parts: truncation error and regularization error. Truncation error measures the difference between the exact Gegenbauer coefficients with $\lambda \sim N$, and those obtained by using the spectral partial sum. This will be investigated in $\S 3$. The regularization error measures the difference between the Gegenbauer expansion using the first few Gegenbauer coefficients with $\lambda \sim N$, and the function itself in a subinterval $[a, b]$, in which the function is assumed analytic. This error is estimated in [6] and we will simply quote the result in $\S 4$. The results are summarized in Theorem 4.3, and some remarks are also given in $\S 4$. Section 5 contains two numerical examples to illustrate our results. In $\S 2$ we collect some useful properties of Gegenbauer polynomials to be used later.

Throughout this paper, we will use $A$ to denote a generic constant or at most a polynomial in the growing parameters, as will be indicated in the text. It may not be the same at different locations.

\section{Preliminaries}

In this section we collect some useful results about the Gegenbauer polynomials, to be used in later sections. We rely heavily on the standardization in Bateman [1].

Definition 2.1. The Gegenbauer polynomial $C_{n}^{\lambda}(x)$, for $\lambda \geq 0$, is defined by

$$
\left(1-x^{2}\right)^{\lambda-\frac{1}{2}} C_{n}^{\lambda}(x)=G(\lambda, n) \frac{d^{n}}{d x^{n}}\left[\left(1-x^{2}\right)^{n+\lambda-\frac{1}{2}}\right],
$$

where $G(\lambda, n)$ is given by

$$
G(\lambda, n)=\frac{(-1)^{n} \Gamma\left(\lambda+\frac{1}{2}\right) \Gamma(n+2 \lambda)}{2^{n} n ! \Gamma(2 \lambda) \Gamma\left(n+\lambda+\frac{1}{2}\right)}
$$

for $\lambda>0$, by

$$
G(0, n)=\frac{(-1)^{n} \sqrt{\pi}}{2^{n-1} n \Gamma\left(n+\frac{1}{2}\right)}
$$

for $\lambda=0$ and $n \geq 1$, and by

$$
G(0,0)=1
$$

for $\lambda=0, n=0$. Notice that by this standardization, $C_{n}^{0}(x)$ is defined by $($ see $[1])$

$$
C_{n}^{0}(x)=\lim _{\lambda \rightarrow 0^{+}} \frac{1}{\lambda} C_{n}^{\lambda}(x)=\frac{2}{n} T_{n}(x), \quad n \geq 1 ; C_{0}^{0}(x)=1,
$$

where $T_{n}(x)$ are the Chebyshev polynomials.

Formula (2.1) is also called the Rodrigues formula [1, page 175].

Under this definition we have, for $\lambda>0$,

$$
C_{n}^{\lambda}(1)=\frac{\Gamma(n+2 \lambda)}{n ! \Gamma(2 \lambda)}
$$

and

$$
\left|C_{n}^{\lambda}(x)\right| \leq C_{n}^{\lambda}(1), \quad-1 \leq x \leq 1 .
$$


The Gegenbauer polynomials are orthogonal with respect to their weight function $\left(1-x^{2}\right)^{\lambda-\frac{1}{2}}$ :

$$
\int_{-1}^{1}\left(1-x^{2}\right)^{\lambda-\frac{1}{2}} C_{k}^{\lambda}(x) C_{n}^{\lambda}(x) d x=\delta_{k, n} h_{n}^{\lambda}
$$

where, for $\lambda>0$,

$$
h_{n}^{\lambda}=\pi^{\frac{1}{2}} C_{n}^{\lambda}(1) \frac{\Gamma\left(\lambda+\frac{1}{2}\right)}{\Gamma(\lambda)(n+\lambda)} .
$$

We will need to use heavily the asymptotics of the Gegenbauer polynomials for large $n$ and $\lambda$. For this we need the well-known Stirling formula

$$
(2 \pi)^{\frac{1}{2}} x^{x+\frac{1}{2}} e^{-x} \leq \Gamma(x+1) \leq(2 \pi)^{\frac{1}{2}} x^{x+\frac{1}{2}} e^{-x} e^{\frac{1}{12 x}}, \quad x \geq 1 .
$$

Lemma 2.2. There exists a constant $A$ independent of $\lambda$ and $n$ such that

$$
A^{-1} \frac{\lambda^{\frac{1}{2}}}{(n+\lambda)} C_{n}^{\lambda}(1) \leq h_{n}^{\lambda} \leq A \frac{\lambda^{\frac{1}{2}}}{(n+\lambda)} C_{n}^{\lambda}(1) .
$$

The proof follows from (2.9) and Stirling's formula (2.10).

We also need the following lemma, which is easily obtained from the Rodrigues formula (2.1).

Lemma 2.3. For any $\lambda \geq 1$ we have

$$
\frac{d}{d x}\left[\left(1-x^{2}\right)^{\lambda-\frac{1}{2}} C_{n}^{\lambda}(x)\right]=\frac{G(\lambda, n)}{G(\lambda-1, n+1)}\left(1-x^{2}\right)^{\lambda-\frac{3}{2}} C_{n+1}^{\lambda-1}(x) .
$$

The proof follows from taking one derivative $\frac{d}{d x}$ on both sides of the Rodrigues formula (2.1), and then using it again on the right-hand side.

Finally, we will need to use the following formula [1, page 176]:

$$
C_{n}^{\mu}(x)=\frac{1}{2(n+\mu)} \cdot \frac{d}{d x}\left[C_{n+1}^{\mu}(x)-C_{n-1}^{\mu}(x)\right],
$$

which is true for all $\mu \geq 0$.

\section{TRUNCATION ERROR IN A SUBINTERVAL}

Consider an arbitrary $L_{1}$ function $f(x)$ defined in $[-1,1]$. Suppose that the first $0 \leq k \leq N$ Gegenbauer coefficients, based upon the Gegenbauer polynomials $C_{k}^{\mu}(x)$ with the weight function $\left(1-x^{2}\right)^{\mu-\frac{1}{2}}$ for any constant $\mu \geq 0$, over the full interval $[-1,1]$, are given:

$$
\hat{f}^{\mu}(k)=\frac{1}{h_{k}^{\mu}} \int_{-1}^{1}\left(1-x^{2}\right)^{\mu-\frac{1}{2}} C_{k}^{\mu}(x) f(x) d x, \quad 0 \leq k \leq N .
$$

We are interested in finding the Gegenbauer expansion of $f(x)$, with $\lambda \sim N$, based on a subinterval $[a, b] \subset[-1,1]$. We start by introducing the local variable $\xi$. 
Definition 3.1. The local variable $\xi$ is defined by

$$
x=x(\xi)=\varepsilon \xi+\delta,
$$

where

$$
\varepsilon=\frac{b-a}{2}, \quad \delta=\frac{b+a}{2} .
$$

Thus, when $a \leq x \leq b$, we have $-1 \leq \xi \leq 1$.

We consider functions $f(x)$ satisfying

Assumption 3.2. $\left|\hat{f}^{\mu}(k)\right| \leq A$ independent of $k$.

We remark that if $f(x)$ is an $L_{1}$ function this assumption is fulfilled.

Since we know the first $N+1$ Gegenbauer coefficients, $\hat{f}^{\mu}(k)$ for $0 \leq k \leq N$, we define the Gegenbauer partial sum

$$
f_{N}^{\mu}(x)=\sum_{k=0}^{N} \hat{f}^{\mu}(k) C_{k}^{\mu}(x) .
$$

Note that $f_{N}^{\mu}(x)$ does not converge fast to $f(x)$ if there exist discontinuities inside the domain.

The function $f(x)$ has also a Gegenbauer expansion in a subinterval $[a, b]$, with $\lambda \sim N$. With $\xi, \varepsilon$ and $\delta$ defined in (3.2)-(3.3), we have

$$
f(\varepsilon \xi+\delta)=\sum_{l=0}^{\infty} \hat{f}_{\varepsilon}^{\lambda}(l) C_{l}^{\lambda}(\xi), \quad-1 \leq \xi \leq 1,
$$

where the Gegenbauer coefficients $\hat{f}_{\varepsilon}^{\lambda}(l)$ are defined by

$$
\hat{f}_{\varepsilon}^{\lambda}(l)=\frac{1}{h_{l}^{\lambda}} \int_{-1}^{1}\left(1-\xi^{2}\right)^{\lambda-\frac{1}{2}} C_{l}^{\lambda}(\xi) f(\varepsilon \xi+\delta) d \xi .
$$

Of course, we do not have $\hat{f}_{\varepsilon}^{\lambda}(l)$ at our disposal, but only an approximation based on the Gegenbauer partial sum $f_{N}^{\mu}(x)$; thus we have

$$
\hat{g}_{\varepsilon}^{\lambda}(l)=\frac{1}{h_{l}^{\lambda}} \int_{-1}^{1}\left(1-\xi^{2}\right)^{\lambda-\frac{1}{2}} C_{l}^{\lambda}(\xi) f_{N}^{\mu}(\varepsilon \xi+\delta) d \xi .
$$

How well do $\hat{g}_{\varepsilon}^{\lambda}(l)$ approximate $\hat{f}_{\varepsilon}^{\lambda}(l)$ ? To answer this question we define:

Definition 3.3. The truncation error is defined by

$$
T E(\lambda, m, N, \varepsilon)=\max _{-1 \leq \xi \leq 1}\left|\sum_{l=0}^{m}\left(\hat{f}_{\varepsilon}^{\lambda}(l)-\hat{g}_{\varepsilon}^{\lambda}(l)\right) C_{l}^{\lambda}(\xi)\right|,
$$

where $\hat{f}_{\varepsilon}^{\lambda}(l)$ are defined by (3.6) and $\hat{g}_{\varepsilon}^{\lambda}(l)$ are defined by (3.7).

The truncation error is the measure of the distance between the true Gegenbauer expansion in the interval $[a, b]$ and its approximation based on the Gegenbauer partial sum in $[-1,1]$.

We first have the following lemma: 
Lemma 3.4. The truncation error can be estimated by

$T E(\lambda, m, N, \varepsilon) \leq \sum_{q=N+1}^{\infty}\left|\hat{f}^{\mu}(q)\right| \sum_{l=0}^{m}\left|\frac{C_{l}^{\lambda}(1)}{h_{l}^{\lambda}} \int_{-1}^{1}\left(1-\xi^{2}\right)^{\lambda-\frac{1}{2}} C_{l}^{\lambda}(\xi) C_{q}^{\mu}(\varepsilon \xi+\delta) d \xi\right|$.

Proof. From (3.6) and (3.7) we have

$$
\hat{f}_{\varepsilon}^{\lambda}(l)-\hat{g}_{\varepsilon}^{\lambda}(l)=\frac{1}{h_{l}^{\lambda}} \int_{-1}^{1}\left(1-\xi^{2}\right)^{\lambda-\frac{1}{2}} C_{l}^{\lambda}(\xi)\left(f(\varepsilon \xi+\delta)-f_{N}^{\mu}(\varepsilon \xi+\delta)\right) d \xi .
$$

Substituting (3.10) into (3.8), recalling (2.7) and

$$
f(\varepsilon \xi+\delta)-f_{N}^{\mu}(\varepsilon \xi+\delta)=\sum_{q=N+1}^{\infty} \hat{f}^{\mu}(q) C_{q}^{\mu}(\varepsilon \xi+\delta),
$$

we obtain (3.9).

For simplicity of notations we denote

$$
F_{q}^{\lambda, l}=\int_{-1}^{1}\left(1-\xi^{2}\right)^{\lambda-\frac{1}{2}} C_{l}^{\lambda}(\xi) C_{q}^{\mu}(\varepsilon \xi+\delta) d \xi .
$$

In order to estimate this term, we start with the following

Lemma 3.5. If we denote

$$
I_{q}^{\lambda, l}=\frac{F_{q}^{\lambda, l}}{G(\lambda, l)}
$$

where $G(\lambda, l)$ is defined by (2.2)-(2.4), then we have the following recursive formula:

$$
I_{q}^{\lambda, l}=\frac{1}{2(q+\mu) \varepsilon}\left[I_{q-1}^{\lambda-1, l+1}-I_{q+1}^{\lambda-1, l+1}\right], \quad \lambda \geq 1, q \geq 1 .
$$

Proof. By the definition of $I_{q}^{\lambda, l}$ in (3.13)-(3.12), we have

$$
\begin{aligned}
I_{q}^{\lambda, l}= & \frac{1}{G(\lambda, l)} \int_{-1}^{1}\left(1-\xi^{2}\right)^{\lambda-\frac{1}{2}} C_{l}^{\lambda}(\xi) C_{q}^{\mu}(\varepsilon \xi+\delta) d \xi \\
= & \frac{1}{G(\lambda, l)} \cdot \frac{1}{2(q+\mu) \varepsilon} \\
& \cdot \int_{-1}^{1}\left(1-\xi^{2}\right)^{\lambda-\frac{1}{2}} C_{l}^{\lambda}(\xi) \frac{d}{d \xi}\left[C_{q+1}^{\mu}(\varepsilon \xi+\delta)-C_{q-1}^{\mu}(\varepsilon \xi+\delta)\right] d \xi \\
= & \frac{1}{2 G(\lambda, l)(q+\mu) \varepsilon} \\
& \cdot \int_{-1}^{1} \frac{d}{d \xi}\left[\left(1-\xi^{2}\right)^{\lambda-\frac{1}{2}} C_{l}^{\lambda}(\xi)\right]\left[C_{q-1}^{\mu}(\varepsilon \xi+\delta)-C_{q+1}^{\mu}(\varepsilon \xi+\delta)\right] d \xi \\
= & \frac{1}{2 G(\lambda-1, l+1)(q+\mu) \varepsilon} \\
& \cdot \int_{-1}^{1}\left(1-\xi^{2}\right)^{\lambda-\frac{3}{2}} C_{l+1}^{\lambda-1}(\xi)\left[C_{q-1}^{\mu}(\varepsilon \xi+\delta)-C_{q+1}^{\mu}(\varepsilon \xi+\delta)\right] d \xi \\
= & \frac{1}{2(q+\mu) \varepsilon}\left[I_{q-1}^{\lambda-1, l+1}-I_{q+1}^{\lambda-1, l+1}\right],
\end{aligned}
$$


where we have used (2.13) for the second equality, integration by parts for the third equality (the boundary terms vanish because of the term $\left(1-\xi^{2}\right)^{\lambda-\frac{1}{2}}$ with $\lambda \geq 1)$, formula (2.12) for the fourth equality, and the definition (3.12)-(3.13) for the last equality.

We can now obtain the following recursive estimate for $I_{q}^{\lambda, l}$ :

Lemma 3.6. The $I_{q}^{\lambda, l}$ defined by (3.13) satisfies the following estimate:

$$
\left|I_{q}^{\lambda, l}\right| \leq \frac{\Gamma(q+\mu+1-j)}{\varepsilon^{j} \Gamma(q+\mu+1)} \max _{q-j \leq p \leq q+j}\left|I_{p}^{\lambda-j, l+j}\right|, \quad j \leq \min (\lambda, q) .
$$

Proof. We use induction on $j$. The estimate is clearly valid for $j=0$. Assume that it is valid for $j=j_{0} \leq \min (\lambda, q)-1$; then

$$
\begin{aligned}
\left|I_{q}^{\lambda, l}\right| \leq & \frac{\Gamma\left(q+\mu+1-j_{0}\right)}{\varepsilon^{j_{0}} \Gamma(q+\mu+1)} \max _{q-j_{0} \leq p \leq q+j_{0}}\left|I_{p}^{\lambda-j_{0}, l+j_{0}}\right| \\
\leq & \frac{\Gamma\left(q+\mu+1-j_{0}\right)}{\varepsilon^{j_{0}} \Gamma(q+\mu+1)} \max _{q-j_{0} \leq p \leq q+j_{0}}\left|\frac{1}{2(p+\mu) \varepsilon}\left[I_{p-1}^{\lambda-j_{0}-1, l+j_{0}+1}-I_{p+1}^{\lambda-j_{0}-1, l+j_{0}+1}\right]\right| \\
\leq & \frac{\Gamma\left(q+\mu+1-j_{0}\right)}{\varepsilon^{j_{0}} \Gamma(q+\mu+1)} \\
& \cdot \frac{1}{2\left(q-j_{0}+\mu\right) \varepsilon} \max _{q-j_{0} \leq p \leq q+j_{0}}\left[\left|I_{p-1}^{\lambda-j_{0}-1, l+j_{0}+1}\right|+\left|I_{p+1}^{\lambda-j_{0}-1, l+j_{0}+1}\right|\right] \\
\leq & \frac{\Gamma\left(q+\mu-j_{0}\right)}{\varepsilon^{j_{0}+1} \Gamma(q+\mu+1)} \max _{q-j_{0}-1 \leq p \leq q+j_{0}+1}\left|I_{p}^{\lambda-j_{0}-1, l+j_{0}+1}\right|,
\end{aligned}
$$

where we have used (3.14) for the second inequality. All other steps are simple inequalities.

This finishes the induction.

From the previous lemma we can get the following estimate:

Lemma 3.7. For $F_{q}^{\lambda, l}$ defined in (3.12) with $\lambda \leq q$ we have the following estimate:

$$
\left|F_{q}^{\lambda, l}\right| \leq A \frac{\Gamma(q-\lambda)}{\varepsilon^{\lambda} \Gamma(q)} \cdot \frac{|G(\lambda, l)|}{|G(0, l+\lambda)|},
$$

where $A$ grows at most as $q^{2 \mu-1}$.

Proof. For simplicity, and without loss of generality, we assume $\lambda$ is an integer. Since $\lambda \leq q$, we can take $j=\lambda$ in (3.15) to arrive at

$$
\left|I_{q}^{\lambda, l}\right| \leq \frac{\Gamma(q+\mu+1-\lambda)}{\varepsilon^{\lambda} \Gamma(q+\mu+1)} \max _{q-\lambda \leq p \leq q+\lambda}\left|I_{p}^{0, I+\lambda}\right| \leq \frac{\Gamma(q-\lambda)}{\varepsilon^{\lambda} \Gamma(q)} \max _{q-\lambda \leq p \leq q+\lambda}\left|I_{p}^{0, I+\lambda}\right| .
$$

By the definition (3.12)-(3.13), we have, for $q-\lambda \leq p \leq q+\lambda$, 


$$
\begin{aligned}
\mid I_{p}^{0, l+\lambda \mid} & =\frac{1}{|G(0, l+\lambda)|}\left|\int_{-1}^{1}\left(1-\xi^{2}\right)^{-\frac{1}{2}} C_{l+\lambda}^{0}(\xi) C_{p}^{\mu}(\varepsilon \xi+\delta) d \xi\right| \\
& \leq \frac{C_{l+\lambda}^{0}(1) C_{p}^{\mu}(1)}{|G(0, l+\lambda)|} \int_{-1}^{1}\left(1-\xi^{2}\right)^{-\frac{1}{2} d \xi} \\
& \leq \frac{\Gamma(l+\lambda)}{(l+\lambda) !} \cdot \frac{\Gamma(p+2 \mu)}{p ! \Gamma(2 \mu)} \cdot \frac{1}{|G(0, l+\lambda)|} \int_{-1}^{1}\left(1-\xi^{2}\right)^{-\frac{1}{2} d \xi} \\
& \leq A \frac{1}{|G(0, l+\lambda)|},
\end{aligned}
$$

where for the second inequality we have used (2.7) and for the third inequality we have used (2.6). Clearly, $A$ is a constant if $\mu \leq \frac{1}{2}$ and $A$ grows at most as $q^{2 \mu-1}$ if $\mu>\frac{1}{2}$. Invoking (3.13) again, we obtain (3.16).

Using Stirling's formula, we can now easily get:

Lemma 3.8. For $l \leq m \leq N$ and $q>N$, we have

$$
\left|F_{q}^{\lambda, l}\right| \leq A \frac{(m+2 \lambda)^{m+2 \lambda}}{(2 \varepsilon \lambda)^{\lambda} m^{m}} \cdot \frac{1}{q^{\lambda}}
$$

where $A$ again grows at most as $(m+\lambda)^{\frac{1}{2}} q^{2 \mu-1}$.

Proof. Starting from (3.16) and using the definition (2.2)-(2.3), we obtain

$$
\begin{aligned}
\left|F_{q}^{\lambda, l}\right| & \leq A \frac{\Gamma(q-\lambda)}{\varepsilon^{\lambda} \Gamma(q)} \cdot \frac{|G(\lambda, l)|}{|G(0, l+\lambda)|} \\
& \leq A \frac{\Gamma(q-\lambda)}{\varepsilon^{\lambda} \Gamma(q)} \cdot \frac{\Gamma\left(\lambda+\frac{1}{2}\right) \Gamma(l+2 \lambda)}{2^{l} l ! \Gamma(2 \lambda) \Gamma\left(l+\lambda+\frac{1}{2}\right)} \cdot 2^{l+\lambda-1}(l+\lambda) \Gamma\left(l+\lambda+\frac{1}{2}\right) \\
& \leq A \frac{\Gamma(q-\lambda)}{\varepsilon^{\lambda} \Gamma(q)} \cdot \frac{\Gamma(\lambda) \Gamma(l+2 \lambda) 2^{\lambda}}{l ! \Gamma(2 \lambda)} \\
& \leq A \frac{\Gamma(q-\lambda)}{\varepsilon^{\lambda} \Gamma(q)} \cdot \frac{\Gamma(\lambda) \Gamma(m+2 \lambda) 2^{\lambda}}{m ! \Gamma(2 \lambda)} \\
& \leq A \frac{(q-\lambda)^{q-\lambda} e^{-(q-\lambda)}}{\varepsilon^{\lambda} q^{q} e^{-q}} \cdot \frac{\lambda^{\lambda} e^{-\lambda}(m+2 \lambda)^{m+2 \lambda} e^{-(m+2 \lambda)} 2^{\lambda}}{m^{m} e^{-m}(2 \lambda)^{2 \lambda} e^{-2 \lambda}} \\
& \leq A \frac{(m+2 \lambda)^{m+2 \lambda}}{(2 \varepsilon \lambda)^{\lambda} m^{m}} \cdot \frac{1}{q^{\lambda}},
\end{aligned}
$$

where we have used (2.2)-(2.3) in the second inequality, the monotonicity with respect to $l$ in the fourth inequality, and Stirling's formula $(2.10)$ for the fifth inequality.

We are now ready for the main theorem of this section:

Theorem 3.9. Let the truncation error be defined in (3.8). Let $\lambda=\alpha \varepsilon N$ and $m=\beta \varepsilon N$ with $0<\alpha, \beta<1$; then 


$$
T E(\alpha \varepsilon N, \beta \varepsilon N, N, \varepsilon) \leq A\left(\frac{(\beta+2 \alpha)^{\beta+2 \alpha}}{2^{\alpha} \alpha^{\alpha} \beta^{\beta}}\right)^{\varepsilon N},
$$

where $A$ grows at most as $N^{1+2 \mu}$. In particular, if $\alpha=\beta<\frac{2}{27}$, then

$$
T E(\alpha \varepsilon N, \alpha \varepsilon N, N, \varepsilon) \leq A q^{\varepsilon N},
$$

where

$$
q=\left(\frac{27 \alpha}{2}\right)^{\alpha}<1
$$

Proof. The theorem follows from (3.9), the Assumption 3.2, (2.11), and (3.17).

\section{REgULARIZATION ERROR AND THE MAIN THEOREM}

The second part of the error, which is called the regularization error and is caused by using a finite Gegenbauer expansion based on a subinterval $[a, b] \subset$ $[-1,1]$, to approximate a function $f(x)$ which is assumed analytic in this subinterval, has been studied in [6]. We will thus just quote the result.

We assume that $f(x)$ is an analytic function on $[a, b]$ satisfying

Assumption 4.1. There exist constants $\rho \geq 1$ and $C(\rho)$ such that, for every $k \geq 0$,

$$
\max _{a \leq x \leq b}\left|\frac{d^{k} f}{d x^{k}}(x)\right| \leq C(\rho) \frac{k !}{\rho^{k}}
$$

This is a standard assumption for analytic functions. The quantity $\rho$ is the distance from $[a, b]$ to the nearest singularity of $f(x)$ in the complex plane (see for example [8]). Let us consider the Gegenbauer partial sum of the first $m$ terms for the function $f(\varepsilon \xi+\delta)$ :

$$
f_{m}^{\lambda, \varepsilon}(\xi)=\sum_{l=0}^{m} \hat{f}_{\varepsilon}^{\lambda}(l) C_{l}^{\lambda}(\xi)
$$

with $\xi, \varepsilon$ and $\delta$ defined by (3.2) and (3.3), and the Gegenbauer coefficients based on $[a, b]$ defined by

$$
\hat{f}_{\varepsilon}^{\lambda}(l)=\frac{1}{h_{l}^{\lambda}} \int_{-1}^{1}\left(1-\xi^{2}\right)^{\lambda-\frac{1}{2}} f(\varepsilon \xi+\delta) C_{l}^{\lambda}(\xi) d \xi .
$$

The regularization error in the maximum norm is defined by

$$
R E(\lambda, m, \varepsilon)=\max _{-1 \leq \xi \leq 1}\left|f(\varepsilon \xi+\delta)-\sum_{l=0}^{m} \hat{f}_{\varepsilon}^{\lambda}(l) C_{l}^{\lambda}(\xi)\right| .
$$

We have the following result for the estimation of the regularization error, when $\lambda \sim m[6]$ : 
Theorem 4.2. Assume $\lambda=\gamma m$, where $\gamma$ is a positive constant. If $f(x)$ is analytic in $[a, b] \subset[-1,1]$ satisfying Assumption 4.1 , then the regularization error defined in (4.4) can be bounded by

$$
R E(\gamma m, m, \varepsilon) \leq A q^{m},
$$

where $q$ is given by

$$
q=\frac{\varepsilon(1+2 \gamma)^{1+2 \gamma}}{\rho 2^{1+2 \gamma} \gamma^{\gamma}(1+\gamma)^{1+\gamma}},
$$

which is always less than 1. In particular, if $\gamma=1$ and $m=\beta \varepsilon N$, where $\beta$ is a positive constant, then

$$
R E(\beta N, \beta N, \varepsilon) \leq A q^{\varepsilon N}
$$

with

$$
q=\left(\frac{27 \varepsilon}{32 \rho}\right)^{\beta}
$$

We can now combine the estimates for truncation errors and regularization errors to obtain the following main theorem of this paper.

Theorem 4.3 (Removal of the Gibbs Phenomenon for the subinterval case of Gegenbauer partial sum). Consider an $L_{1}$ function $f(x)$ on $[-1,1]$, which is analytic in a subinterval $[a, b] \subset[-1,1]$ and satisfies Assumption 4.1. Assume that the first $N+1$ Gegenbauer coefficients

$$
\hat{f}^{\mu}(k)=\frac{1}{h_{k}^{\mu}} \int_{-1}^{1}\left(1-x^{2}\right)^{\mu-\frac{1}{2}} C_{k}^{\mu}(x) f(x) d x,
$$

for $\mu \geq 0$, are known. Let $\hat{g}_{\varepsilon}^{\lambda}(l), 0 \leq l \leq m$, be the Gegenbauer expansion coefficients, defined in (3.7), based on the subinterval $[a, b]$, of the Gegenbauer partial sum $f_{N}^{\mu}(x)$ in (3.4). Then for $\lambda=m=\beta \varepsilon N$ with $\beta<\frac{2}{27}$, we have

$$
\max _{-1 \leq \xi \leq 1}\left|f(\varepsilon \xi+\delta)-\sum_{l=0}^{m} \hat{g}_{\varepsilon}^{\lambda}(l) C_{l}^{\lambda}(\xi)\right| \leq A\left(q_{T}^{\varepsilon N}+q_{R}^{\varepsilon N}\right),
$$

where

$$
q_{T}=\left(\frac{27 \beta}{2}\right)^{\beta}<1, \quad q_{R}=\left(\frac{27 \varepsilon}{32 \rho}\right)^{\beta}<1,
$$

and $A$ grows at most as $N^{1+2 \mu}$.

Proof. Just combine the results of Theorems 3.9 and 4.2 .

We now make two remarks.

Remark 4.3. Comparing with the Legendre case in [6], we can see that the current proof is less sharp (missing a factor of $\frac{1}{\varepsilon}$ in the truncation error $q_{T}$ ). The main loss in this sharpness is in the estimate (3.15).

Remark 4.4. No attempt has been made to optimize the parameters. 


\section{NumericAl RESUltS}

In this section we give two numerical examples to illustrate our result. We will test Chebyshev series because these are used most often in practice. Notice that the Chebyshev polynomials are just Gegenbauer polynomials with $\mu=0$ modulo a constant: $T_{k}(x)=\frac{k}{2} C_{k}^{0}(x)$.

Example 5.1. We take the simple step function

$$
f(x)= \begin{cases}1 & \text { if } a \leq x \leq b \\ 0 & \text { otherwise }\end{cases}
$$

and assume that we know the first $N+1$ Chebyshev coefficients of $f(x)$ :

$$
\hat{f}^{0}(k)=\frac{2}{\pi c_{k}} \int_{-1}^{1}\left(1-x^{2}\right)^{-\frac{1}{2}} T_{k}(x) f(x) d x, \quad 0 \leq k \leq N,
$$

where

$$
c_{k}= \begin{cases}2 & \text { if } k=0 \\ 1 & \text { if } k \geq 1\end{cases}
$$

We then form the Chebyshev partial sum

$$
f_{N}^{0}(x)=\sum_{k=0}^{N} \hat{f}^{0}(k) T_{k}(x)
$$

and then compute the approximate Gegenbauer expansion coefficient based on the subinterval $[a, b]$ defined by (3.7):

$$
\hat{g}_{\varepsilon}^{\lambda}(l)=\frac{1}{h_{l}^{\lambda}} \int_{--1}^{1}\left(1-\xi^{2}\right)^{\lambda-\frac{1}{2}} C_{l}^{\lambda}(\xi) f_{N}^{0}(\varepsilon \xi+\delta) d \xi .
$$

With these Gegenbauer coefficients, we can finally compute the uniformly accurate approximation on $[a, b]$ defined by

$$
g_{m}^{\lambda}(x)=\sum_{l=0}^{m} \hat{g}_{\varepsilon}^{\lambda}(l) C_{l}^{\dot{\lambda}}(\xi)
$$

Numerical experiments (for various functions) seem to indicate that

$$
m=0.1 \varepsilon N, \quad \lambda=0.2 \varepsilon N
$$

are good choices. Notice that in our proof we did not attempt to optimize these parameters. For consistency we will use (5.7) for both examples.

For this special function (5.1), there is no regularization error. Hence all we see is the truncation error. In Fig. 1, top, we show the errors of a middle subinterval $[a, b]=[-0.5,0.5]$, and in Fig. 1, bottom, we show that of a onesided subinterval $[a, b]=[0,1]$. We can clearly see good convergence for both cases. 

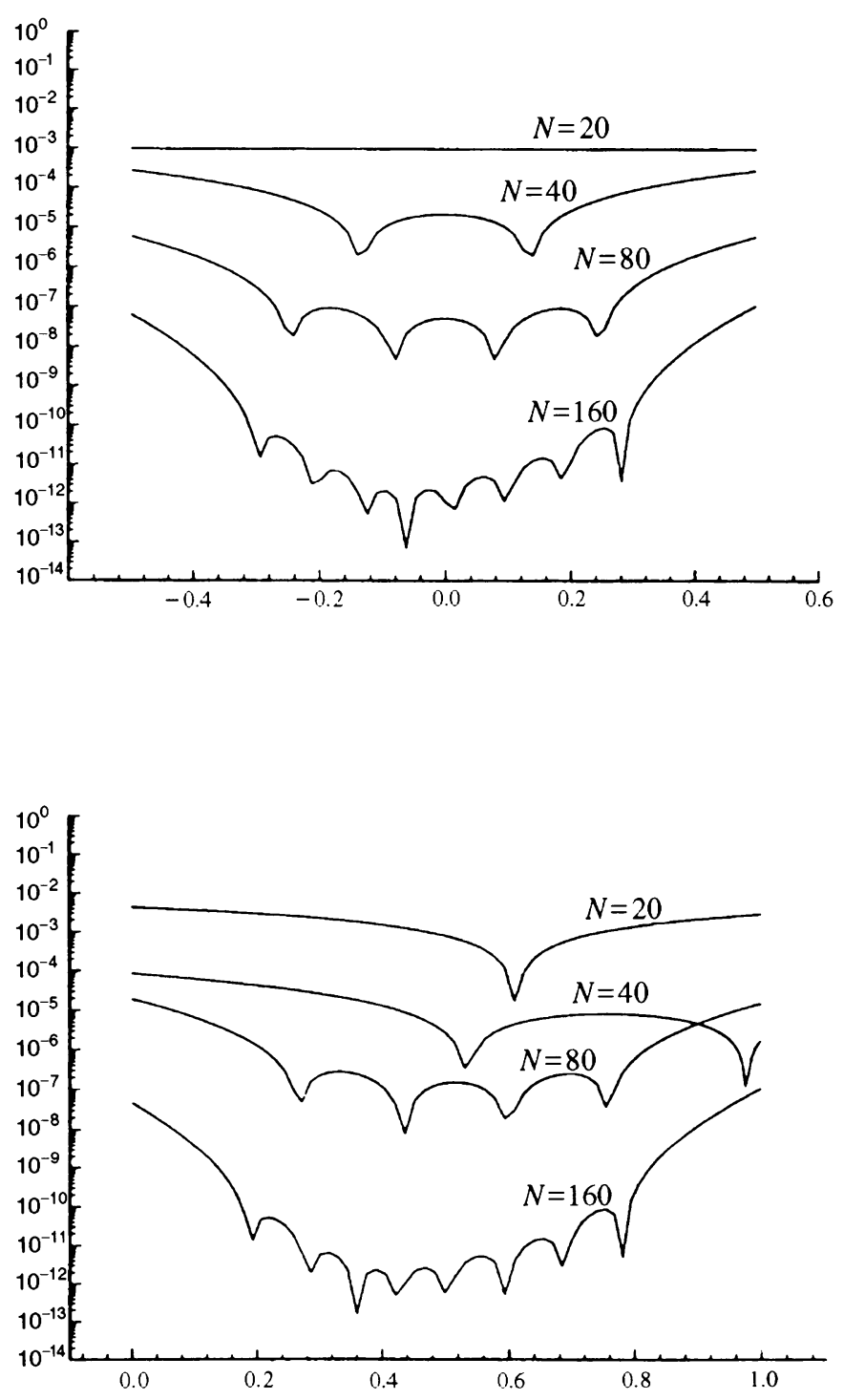

FIGURE 1. Errors in log scale, $f(x)$ defined by $(5.1)$. $[a, b]=$ $[-0.5,0.5]$ (top) and $[a, b]=[0,1]$ (bottom). $\lambda=0.2 \varepsilon N$ and $m=0.1 \varepsilon N . N=20,40,80,160$

Since there is no regularization error for this example, and the truncation error is smaller for small $m$, we also plot the errors for $m=1$ and $\lambda=0.2 \varepsilon N$ in Fig. 2. We can see that the errors are now much smaller than those in Fig. 1. Of course for general functions, regularization errors must balance with truncation errors, so we cannot expect $m=1$ to work for the general case. 

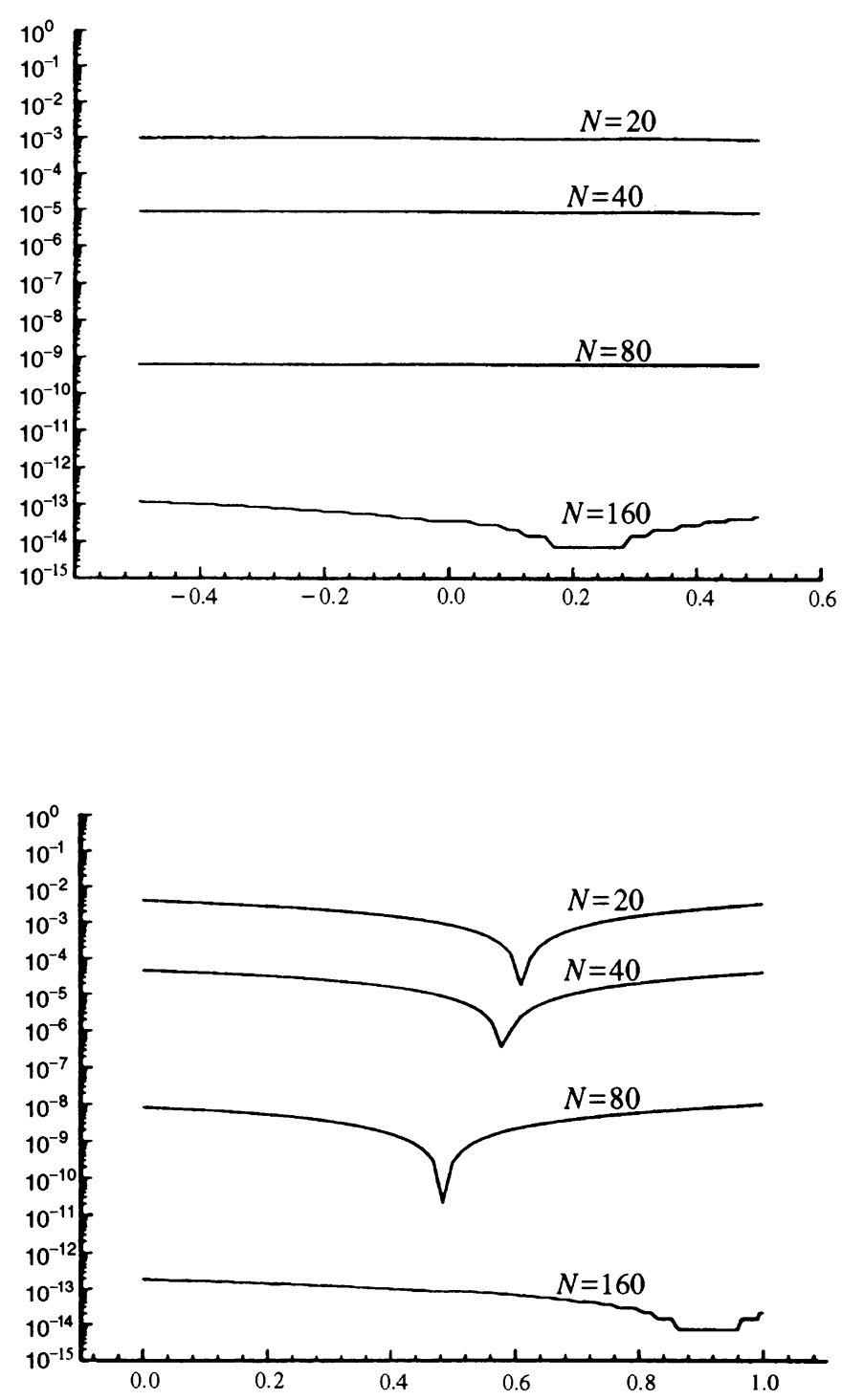

Figure 2. Errors in log scale, $f(x)$ defined by $(5.1) .[a, b]=$ $[-0.5,0.5]$ (top) and $[a, b]=[0,1]$ (bottom). $\lambda=0.2 \varepsilon N$ and $m=1 . N=20,40,80,160$

Example 5.2. In the second example we take the following function:

$$
f(x)= \begin{cases}\sin (\cos (x)) & \text { if } a \leq x \leq b \\ 0 & \text { otherwise }\end{cases}
$$

Again, we assume that we know the first $N+1$ Chebyshev coefficients of $f(x)$ defined by (5.2). 

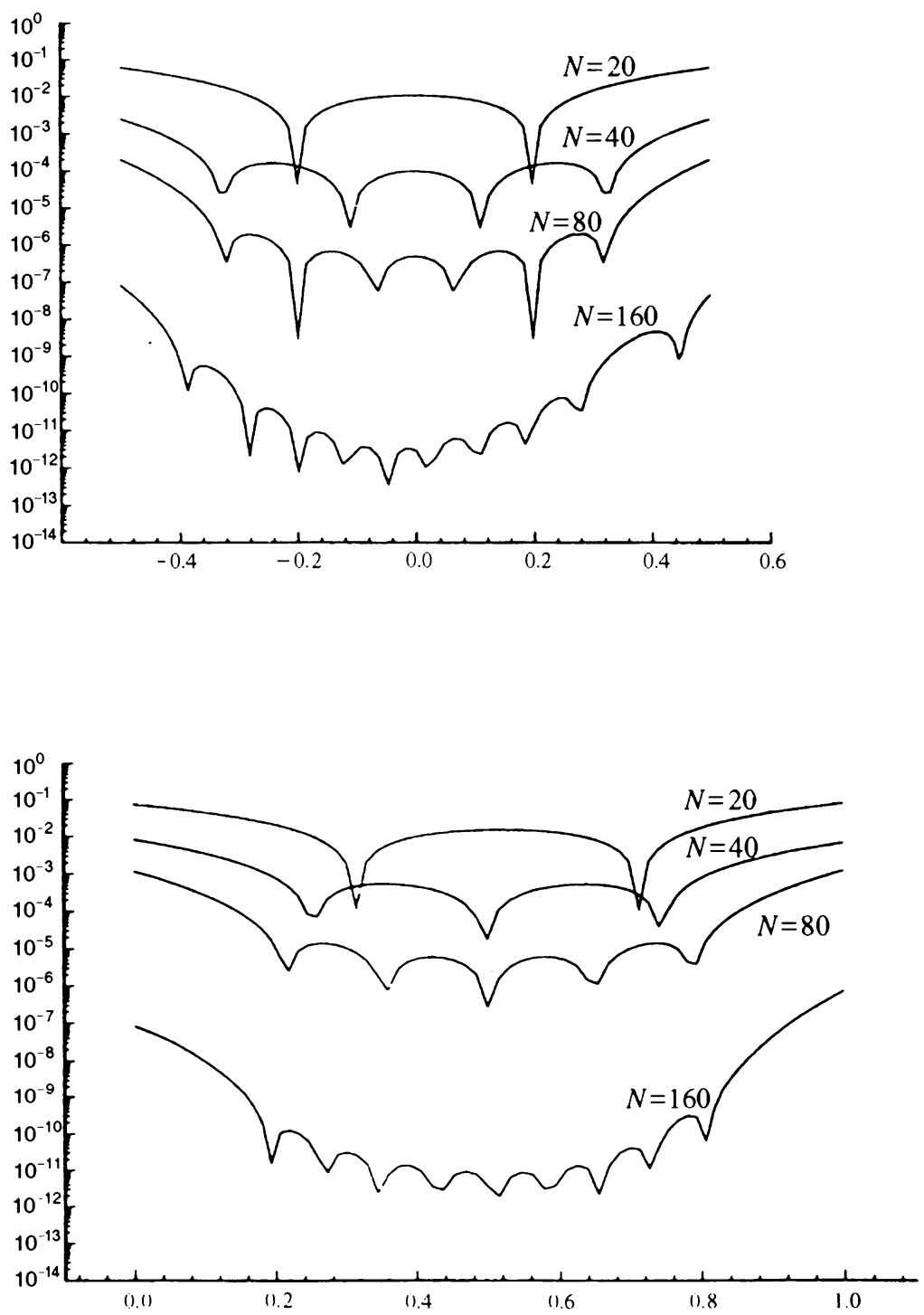

Figure 3. Errors in log scale, $f(x)$ defined by $(5.8)$. $[a, b]=$ $[-0.5,0.5]$ (top) and $[a, b]=[0,1]$ (bottom). $\lambda=0.2 \varepsilon N$ and $m=0.1 \varepsilon N . N=20,40,80,160$

This time both truncation error and regularization error exist. We again pick two cases with middle as well as one-sided subintervals. From Fig. 3 we can see similar results as in the previous example, Fig. 1.

These examples illustrate well the good convergence behavior of our approach. 


\section{BIBLIOGRAPHY}

1. H. Bateman, Higher transcendental functions, Vol. 2, McGraw-Hill, New York, 1953.

2. C. Canuto, M. Y. Hussaini, A. Quarteroni, and T. A. Zang, Spectral methods in fluid dynamics, Springer-Verlag, Berlin and New York, 1988.

3. D. Gottlieb and S. Orszag, Numerical analysis of spectral methods: Theory and applications, SIAM-CBMS, Philadelphia, PA, 1977.

4. D. Gottlieb, C.-W. Shu, A. Solomonoff, and H. Vandeven, On the Gibbs phenomenon I: Recovering exponential accuracy from the Fourier partial sum of a nonperiodic analytic function, J. Comput. Appl. Math. 43 (1992), 81-92.

5. D. Gottlieb and C.-W. Shu, Resolution properties of the Fourier method for discontinuous waves, Comput. Methods Appl. Mech. Engrg. 116 (1994), 27-37.

6. __ On the Gibbs phenomenon III: Recovering exponential accuracy in a sub-interval from the spectral partial sum of a piecewise analytic function, ICASE Report No. 93-82, NASA Langley Research Center, 1993; SIAM J. Numer. Anal. (to appear).

7. I. Gradshteyn and I. Ryzhik, Tables of integrals, series, and products, Academic Press, New York, 1980.

8. F. John, Partial differential equations, Springer-Verlag, Berlin and New York, 1982.

Division of Applied Mathematics, Brown University, Providence, Rhode Island 02912 E-mail address, D. Gottlieb: dig@cfm.brown.edu

E-mail address, C.-W. Shu: shu@cfm.brown.edu 